\title{
Die Suche nach altem Wald in der Schweiz
}

\author{
Rita Bütler \\ Markus Bolliger \\ Brigitte Commarmot
}

\author{
Eidgenössische Forschungsanstalt für Wald, Schnee und Landschaft WSL, Standort Lausanne (CH)* \\ Bundesamt für Umwelt BAFU (CH) \\ Eidgenössische Forschungsanstalt für Wald, Schnee und Landschaft WSL (CH)
}

\begin{abstract}
Die Suche nach altem Wald in der Schweiz
Altwälder und Altwaldbestände haben vielfältige Werte: biologische, wissenschaftliche, sozioökonomische, ästhetische, spirituelle und kulturelle. Zugleich sind sie in Mitteleuropa selten. Zurzeit gibt es in der Schweiz keinen Gesamtüberblick über das Vorkommen und die Ausdehnung alter Wälder. Wir schlagen eine Definition für erhaltenswerte Altwälder anhand von zehn qualitativen Merkmalen vor: 1) hohes Baumalter, 2) grosser Gesamtvorrat, 3) heterogener Bestandesaufbau mit späten Entwicklungsstadien, 4) Vorkommen natürlicher Prozesse und Störungen, 5) grosse Totholzmengen (mind. $30 \mathrm{~m}^{3} / \mathrm{ha}$ ) in unterschiedlichen Dimensionen und Zersetzungsstadien, 6) naturnahe Bestockung, 7) natürliches Artenspektrum, 8) letzte Nutzung vor mindestens 50 Jahren oder seither nur noch sehr extensive Nutzung, 9) lange Waldkontinuität, 10) Minimalfläche ca. 30 ha für Altwälder und 1 ha für Altwaldbestände. Bei der Suche nach erhaltenswerten Objekten sollen auch quantitative Merkmale aus dem Schweizerischen Landesforstinventar und anderen Quellen genutzt werden. Wir rufen alle Fachleute dazu auf, erhaltenswerte alte Wälder zu identifizieren, Bevölkerung und Entscheidungsträger über ihren Wert zu informieren und sie als einzigartiges Naturerbe entsprechend zu schützen.
\end{abstract}

Keywords: old-growth forest, conservation, biodiversity, forest management doi: $10.3188 /$ szf.2015.0067

*EPFL, Station 2, CH-1015 Lausanne, E-Mail: rita.buetler@wsl.ch
G ibt man bei Google den Begriff «alter Wald» ein, stösst man als Erstes auf Tolkiens Buch «Herr der Ringe», in dem uralte, verwunschene und für unerwünschte Eindringlinge gefährliche Wälder mit eigensinnigen, geheimnisvollen Bäumen eine wichtige Rolle spielen. Als zweiter Eintrag erscheint dann die Meldung «14000 Jahre alter Wald in Zürich gefunden», wo auf den einzigartigen Fund von 14000 Jahre alten Baumstrünken in einer Baugrube in Zürich im Jahr 2013 hingewiesen wird.

Wir verstehen unter alten Wäldern oder Altwäldern (engl. «old-growth forests») naturnah bestockte Wälder, die sich über eine längere Periode (i.d.R. mindestens eine Baumgeneration) weitgehend unbeeinträchtigt von menschlichen Eingriffen entwickeln konnten und auch nicht in jüngerer Zeit durch eine grossflächige Störung, zum Beispiel Windwurf, in ihr Pionierstadium zurückgeworfen wurden. Altwälder erkennt man deshalb an ihrer ungleichen Altersstruktur, am hohen Durchschnittsalter, am Vorkommen von einigen biologisch sehr alten Bäumen sowie an grösseren Mengen von stehendem (Dürrständer) und liegendem Totholz in allen Zersetzungsstadien. Am besten lassen sich Altwälder mit qualitativen Merkmalen definieren (Tabelle 1). Quantitative Merkmale, wie der Totholz- oder der Lebendvorrat müssen jeweils spezifisch für bestimmte Waldtypen und Standorte hergeleitet werden.

Die Merkmale von Altwäldern entsprechen weitgehend denjenigen von Natur- oder Urwäldern. Altwälder müssen aber nicht zwingend unbewirtschaftet sein. Umgekehrt ist nicht jeder Urwald ein alter Wald: D.h. ein Urwaldrelikt, das durch einen Sturm oder ein Feuer so stark beeinträchtigt wurde, dass die Waldentwicklung praktisch auf der gesamten Fläche in ein Jungwaldstadium zurückgeworfen wurde, braucht erst wieder mindestens eine Baumgeneration, um als Altwald gelten zu können.

Altwälder sind typischerweise ungleichmässig aufgebaut. Sie sind gekennzeichnet durch eine grosse Vielfalt an dünnen, mittelstarken und dicken Bäumen in allen möglichen Zuständen (lebende Bäume, Dürrständer, Baumstrünke, liegende Stämme und Äste in unterschiedlichen Dimensionen und Abbaustadien). Baumgruppen, die sich in unterschiedlichen Stadien der Entwicklung befinden, bilden ein räumliches Mosaik, das insgesamt den ganzen Entwicklungszyklus eines Waldes abdeckt. Dadurch ent- 
steht eine Kontinuität an Habitat- und Strukturelementen, welche für das langfristige Überleben diverser Waldarten und Organismengruppen von Bedeutung sind (z.B. Scheidegger et al 2015, dieses Heft). Damit diese Kontinuität langfristig gewährleistet ist und die Altwälder selbst bei seltenen grösseren Ereignissen nicht vollständig zerstört werden, muss deren Fläche eine gewisse Grösse aufweisen. Diese Mindestgrösse ist abhängig vom Waldtyp, vom Standort sowie von der Stärke und der Häufigkeit von Störungen. Bei Schattenbaumarten mit kleinflächiger natürlicher Lückendynamik genügt eine Fläche von etwa 30 bis 50 ha (Korpel' 1995), bei Baumarten, die mehr Licht und grössere Lücken zu ihrer Verjüngung benötigen, kann sie mehrere hundert oder bis über tausend Hektaren gross sein. Je grösser die Fläche von Altwäldern ist, desto grösser ist meistens deren langfristiger ökologischer Wert.

Als Altwaldbestände bezeichnen wir Bestände, die sich in einem späten Stadium der Entwicklung befinden und in denen Einzelbäume nicht nur im Wettbewerb um Raum, Licht und Nährstoffe absterben, wie dies in jungen Wäldern der Fall ist, sondern immer mehr auch infolge von Pilz-, Insektenbefall oder Wind, welche mit zunehmendem Alter der Bäume als Mortalitätsursache an Bedeutung gewinnen. Solche Bestände weisen einerseits viele Elemente von Altwäldern auf wie grosse und alte Bäume, hohe Vorräte an stehendem und liegendem Totholz und eine erhöhte Strukturvielfalt. Andererseits und im Unterschied zu Altwäldern, in denen alle Entwicklungsstadien gleichzeitig nebeneinander vertreten sind, durchlaufen die kleineren Altwaldbestände als Ganzes einen Entwicklungszyklus; d.h. sie üben die typischen «Altwaldfunktionen» nur über einen beschränkten Zeitraum aus. Trotzdem sind auch solche kleine Flächen («Altholzinseln») sehr wertvoll als Reliktstandorte für gefährdete Arten und als sogenannte Trittsteinbiotope mit Vernetzungsfunktion zwischen grösseren Altwäldern.

\section{Alte Wälder haben eine grosse Bedeutung}

Der Wert von alten Wäldern ist vielfältig: biologisch, wissenschaftlich, soziö̈konomisch, geschichtlich, ästhetisch, spirituell und kulturell. Alte Wälder sind Rückzugsgebiete für spezialisierte Waldarten und besonders Urwaldreliktarten, spielen eine wichtige Rolle im Kohlenstoffkreislauf und für die $\mathrm{CO}_{2}$-Bindung, sind Referenzobjekte zur Bewertung der Naturnähe von Wirtschaftswäldern, Studienobjekte zum besseren Verständnis von natürlichen Prozessen und zur Gewinnung von waldbaulichen Erkenntnissen, Bildungsmedium für Umwelt-, Erdund Geisteswissenschaften, Gegenstand und Inspirationsquelle für Dichter und Maler aller Epochen,
Merkmal

Alters- und Bestandesstruktur

ungleichmässig

Hohes Baumalter

Grosser Gesamtvorrat, grosse

Grundfläche

Natürliche Prozesse

Totholz

Naturnahe Bestockung

Artenspektrum,

Urwaldreliktarten

Nutzungsgeschichte

Waldkontinuität

Minimalfläche

\section{Umschreibung}

Die Bestände sind ungleichmässig aufgebaut. Die Verjüngung findet stetig statt. Dadurch grosse Streuung von Baumalter und-durchmesser auf kleiner Fläche. Mosaikartige Mischung verschiedener Entwicklungsstadien. Späte Entwicklungsstadien, insbesondere das Zerfallsstadium, sind stark vertreten.

Zahlreiche alte (älter als die baumartenspezifische Umtriebszeit) und dicke Bäume. Das Durchschnittsalter der herrschenden Baumart entspricht mindestens der halben maximalen Lebenserwartung der Art. Einzelne Bäume sind nahe an ihrem biologischen Maximalalter (je nach Baumart $>200$ bis $>600$ Jahre).

Die Biomasse ist gross und weist über grössere Flächen nur geringfügige Schwankungen auf (Lebendvorrat von $>500 \mathrm{~m}^{3} / \mathrm{ha}$, z.B. für Fichten-, Tannen- und Buchenurwälder in den Karpaten, oder Grundfläche von >35 m²/ha, z.B. für Buchen- und Buchenmischurwälder in den Transkarpaten und in Polen).

Vitalitätsverluste und dichteunabhängige (d.h. nicht konkurrenzbedingte) Mortalität in der Oberschicht. Kleinflächige Störungen, z.B. durch Insektenbefall, Windwurf oder Schneebruch. Freie Waldentwicklung mit kleinflächiger Lückendynamik.

Grosse Mengen an Totholz (mehr als ca. 30 m³/ha oder ab dem Erreichen von ökologischen Schwellenwerten; Müller \& Bütler 2010) in unterschiedlichen Zersetzungsstadien und Dimensionen, insbesondere auch in Form von dicken stehenden und liegenden toten Bäumen.

Bestockung mit standortheimischen Baumarten, vorwiegend aus natürlicher Verjüngung (autochthones Erbgut). Beimischung standortfremder, gepflanzter Baumarten höchstens in geringem Umfang ( $<10 \%$ der Stammzahl). Natürliches Artenspektrum. Komplexe trophische Beziehungen und Nahrungsnetze sind vorhanden. Vorkommen von Urwaldreliktarten (Müller et al 2005).

Letzte Nutzung liegt mindestens 50 Jahre zurück, oder der Bestand wurde höchstens noch schwach genutzt. Spuren anthropogener Nutzungen wie Strünke gefällter Bäume oder Beweidungsspuren fehlen deshalb weitgehend. Standort war, soweit bekannt, immer mit Wald bestockt. Keine Zwischennutzung, z.B. als Weide oder Acker. Dadurch Vorkommen von Arten, die auf lange Kontinuität des Waldes angewiesen sind. (z.B. Winter et al 2015, Scheidegger et al 2015; beide dieses Heft).

30 ha für Altwälder bzw. 1 ha für Altwaldbestände («Altholzinseln»; Jakoby et al 2010, Müller et al 2012).

Tab 1 Merkmale zur Identifizierung von Altwäldern (basierend auf Wirth et al 2009). Im Idealfall treffen alle Merkmale zu, mindestens aber muss ein Wald naturnah bestockt und überdurchschnittlich alt und totholzreich sein, um als Altwald gelten zu können. 
La Pierreuse (Waadt)

Scatlè (Graubünden)

Derborence (Wallis)

St-Jean (Bern)

Bödmerenwald (Schwyz)

Tamangur (Graubünden)

Aletschwald (Wallis)

Dell'Arena, Valle di Vergeletto

(Tessin)
5349 ha Wald unter Prozessschutz, 1400 m bis Waldgrenze. Schneeheide-Bergföhrenwald, Steinrosen-Bergföhrenwald, Lärchen-Arvenwald, Lärchen-Fichtenwald, Erdseggen-Engadinerföhrenwald.

621 ha Naturwaldreservat, 1100 m bis Waldgrenze. Wollreitgras-Tannen-Fichtenwald, Schachtelhalm-TannenFichtenwald, Hochstauden-Tannen-Fichtenwald, Arven-Föhren-Wald.

24 ha Naturwaldreservat, 1510 bis 2015 m ü. M. Torfmoos-Fichtenwald, Alpendostflur mit Fichte. Untersuchungen zu Waldbau und Ertragskunde (Hillgarter 1971), Baumartenzusammensetzung und Absterbeprozesse (Götz 2001). 22.3 ha Naturwaldreservat, 1430 bis 1650 m ü. M. Schneeheide-Bergföhrenwald, Alpendost-Fichten-Tannenwald. 8.7 ha Naturwaldreservat, 1300 bis $1400 \mathrm{~m}$ ü. M. Ahorn-Buchenwald.

Reservat 550 ha, davon 411 ha Naturwaldreservat, 1400 bis 1700 m ü.M. Torfmoos-Fichtenwald, Alpendostflur mit Fichte, Zwergbuchs-Fichtenwald, Steinrosen-Bergföhrenwald. Sehr gut erforscht. Grösster Fichtenwald mit Urwaldcharakter im Alpenraum. Wurde nie beweidet (Karst). Älteste Fichte 484 Jahre. Ausserordentliche Biodiversität. Seit der letzten Eiszeit ohne Unterbrechung bewaldet (maximale Habitatkontinuität). Viele Urwaldreliktarten, z.B. sehr seltene Flechten wie Usnea longissima, Heterodermia speciosa und Parmelia taylorensis. Grosse Artenvielfalt, z.B. 256 Moosarten, 26 Moosarten auf fingernagelgrossem Moospolster. 75\% aller in der Schweiz bekannten Kelchflechten und -pilzen (Caliciales) kommen im Bödmerenwald vor (Gabriel 2001, Kälin 1997, Stiftung Urwaldreservat Bödmeren 2005).

86 ha Naturwaldreservat, 2100 bis 2300 m ü. M. Lärchen-Arvenwald. Höchster Arvenwald Europas. Nur 750 mm Jahresniederschlag und sehr tiefe Temperaturen im Winter (nicht selten unter $-30^{\circ} \mathrm{C}$ ). Früher Nutzung als Waldweide. Höchste genetische Vielfalt der Arvenwälder der Alpen (Höhn et al 2009).

350 ha Naturwaldreservat, 1440 bis 2330 m ü. M. Lärchen-Arvenwald, Torfmoos-Fichtenwald. 1000 Jahre alte Arven, aber früher stark beweidet.

177 ha Naturwaldreservat, 1100 bis 1700 m ü. M. Buchen-Tannenwald, Weisstannen-Fichtenwald.

Tab 2 Porträts einiger alter Wälder der Schweiz.

Ruhezone und Energiequelle für den gestressten Büromenschen und erlauben Begegnungen mit Vorfahren und Geschichte.

Die internationale Initiative für ein UNESCOWeltnaturerbe «Buchenurwälder und alte Buchenwälder Europas» ist Ausdruck des steigenden Interesses an alten Wäldern auf unserem Kontinent. Auch zum Beispiel für die Entwicklung von Kriterien und Indikatoren für eine nachhaltige Waldwirtschaft oder für Forschungsfragen hat das Interesse an Naturwäldern in den letzten zwei Jahrzehnten stark zugenommen.

Klimawandel, massive $\mathrm{CO}_{2}$-Emissionen, die Energiewende sowie der weltweite Rückgang der Artenvielfalt werfen neue Fragen auf, für die alte Wälder als Referenzsysteme und Studienobjekte noch mehr an Bedeutung gewinnen. Die Untersuchung beispielsweise von Veränderungen im Konkurrenzverhalten oder der Koexistenz von Baumarten liefern Grundlagen für den Waldbau. Alte Wälder spielen zudem eine wichtige Rolle als Generhaltungsgebiete.

Bei der Quantifizierung von Ökosystemleistungen wird der Existenz- und Optionswert von Altwäldern beziehungsweise der darin lebenden Organismen bereits ökonomisch bewertet. Der Wert des Madagaskar-Immergrüns, welches die Grundlage für Krebsmedikamente in Europa darstellt, wurde im Nationalpark Masoala in Madagaskar auf 1.6 Mio. US-Dollar geschätzt (European Communities 2008). Auch Altwälder in der Schweiz beherbergen ökonomisch wichtige Arten. So hat zum Beispiel die Weleda AG in Arlesheim Interesse an einer Sammel- bewilligung für die auf der Roten Liste stehende Lungenflechte Lobaria pulmonaria, aus der ein Hustensirup hergestellt wird (Mühlethaler 2006). Nennenswert sind auch die $\mathrm{CO}_{2}$-Emissionszertifikate für gewisse Waldreservate in der Schweiz.

\section{Altwälder sind in Mitteleuropa selten}

Altwälder sind in den gemässigten Zonen Europas selten. Ur- und Naturwaldreste gibt es heute vor allem noch in den Gebirgszügen Ost- und Südosteuropas (z.B. Karpaten, Dinarische und Albanische Alpen, Balkan), wo zu Beginn des 20. Jahrhunderts noch ausgedehnte Urwälder vorkamen. Aber auch in den Alpen haben kleinere Urwaldreste überdauert. Insgesamt wird der Anteil von Ur- und Naturwäldern im gemässigten Europa auf circa $0.4 \%$ der Waldfläche geschätzt (Parviainen 2005). Die meisten derartigen Urwaldrelikte sind weniger als 100 ha gross; zusammenhängende Flächen von mehr als 1000 ha sind sehr selten. Beispiele sind der Buchenurwald in UholkaShyrokyi Luh (Transkarpatien, Ukraine) mit einer Fläche von 8800 ha, der Buchenurwald Izvoarele Nerei (südliche Karpaten, Rumänien) mit 5253 ha und der Mischwald Białowieża (Polen) mit 4747 ha.

Auch in der Schweiz gibt es noch kleinflächige Ur- und Naturwaldreste. Der wohl bekannteste ist der Bödmerenwald (Schwyz), der grösste Gebirgsfichtennaturwald in Europa (Tabelle 2), von dem zurzeit 411 ha als Naturwaldreservat unter Prozessschutz stehen. Kleinere Reste sind in Derborence (22.3 ha, 
Wallis) und in Scatlè (24 ha, Graubünden) zu finden. Die meisten Altwälder in der Schweiz zeigen irgendwelche Spuren einstiger Nutzung, sei es als Waldweide oder zur Holzgewinnung.

\section{Auf der Suche nach alten Wäldern und Altwaldbeständen}

Angesichts der grossen Bedeutung von alten Wäldern und ihrer Seltenheit ist es wichtig, erhaltenswerte Altwälder und -bestände zu identifizieren und zu schützen. In der Schweiz gibt es kein nationales Inventar alter Wälder. Das Wissen über Existenz, Verteilung und Grösse alter Wälder ist nicht gebündelt vorhanden. Das Bundesamt für Umwelt hat zwar in den letzten Jahren eine nationale Datenbank aufgebaut, in der die geografischen Daten aller kantonalen Waldreservate enthalten sind: Das Alter und die Geschichte der darin geschützten Waldbestände sind aber darin nicht erfasst. Ausserdem kommen viele tatsächlich alte Wälder ausserhalb der Reservate vor und fehlen in dieser Datenbank. Möchte man sich einen nationalen Gesamtüberblick verschaffen, müsste gezielt auf die Kenntnisse von lokal tätigen Forstleuten zurückgegriffen werden.

Neben Bestandeskarten und Wirtschaftsplänen sind auch Einzelstudien zu bestimmten Wäldern (z.B. Kälin 1997, Stiftung Urwaldreservat Bödmeren 2005) und Untersuchungen zu einzelnen Altwaldmerkmalen (z.B. Herrmann et al 2012) wertvolle Quellen zum Aufspüren erhaltenswerter alter Wälder. Für gewisse Gebiete wurden ausführliche Forschungen in Publikationsreihen von naturforschenden Gesellschaften publiziert. Das Projekt «Forschung und Wirkungskontrolle in Schweizer Naturwaldreservaten», eine Fortsetzung der Waldreservatsforschung der Eidgenössischen Technischen Hochschule (ETH) seit 1948, liefert laufend neue Erkenntnisse aus diesen Reservaten (z.B. Brang et al 2011, Heiri et al 2012).

Auf nationaler Ebene liefert das Schweizerische Landesforstinventar (LFI) ebenfalls Anhaltspunkte zum Auffinden alter Wälder. Beispiele solcher Indikatoren sind die LFI-Merkmale Bestandesalter, Anzahl Giganten (Bäume mit Brusthöhendurchmesser [BHD] $>80 \mathrm{~cm}$ ), Vorrat und Grundfläche, Totholzmenge, Entwicklungsstufe und Zeitdauer seit dem letzten Eingriff. Durch eine geeignete Kombination dieser Merkmale können LFI-Stichproben identifiziert werden, die bestimmten Kriterien für Altwälder entsprechen. Allerdings beschränkt sich die Aussage auf die einzelne Stichprobe mit sehr kleiner Fläche (200 m² für Bäume mit BHD $\geq 12 \mathrm{~cm}$ und $500 \mathrm{~m}^{2}$ für $\mathrm{BHD} \geq 36 \mathrm{~cm}$ ). Man kann daraus nicht auf die Grösse des Bestandes mit den entsprechenden Merkmalen schliessen. Dennoch lassen sich mit LFI-Daten Schwerpunktregionen finden (Brändli et al 2011), in denen man die Suche nach Altwäldern zum Beispiel mittels lokaler Umfragen intensivieren könnte.

Bedingt durch die lange Tradition eines auf nachhaltige Holzproduktion ausgerichteten Waldbaus beruhen die bei den Forstdiensten vorhandenen Daten zu alten Wäldern und Beständen meist auf waldbaulichen Kriterien und nur selten auf ökologischen Merkmalen. Bei der Waldinventur werden Bestände oft anhand des mittleren Durchmessers der 100 dicksten Bäume pro Hektare $\left(\mathrm{d}_{\mathrm{dom}}\right)$ in Entwicklungsstufen ${ }^{1}$ eingeteilt. Meist endet diese Klassifikation beim «starken Baumholz» (manchmal als «Altholz» bezeichnet), wo die dicksten Bäume einen BHD von $>50 \mathrm{~cm}$ erreichen (z.B. Schütz 2003). Diese Bestände werden dann in der Regel für die «Endnutzung» beziehungsweise Verjüngung vorgesehen. Auf den meisten Standorten können jedoch viele Baumarten erheblich älter und dicker werden, als die übliche waldbauliche Skala vermuten lässt. Es ist wichtig, sich der auf die Holzproduktion ausgerichteten Zielsetzung der gängigen forstlichen Definition bewusst zu sein: Der Schweizer Wald mag aus Sicht der Holzproduktion heute vielerorts als überaltert beurteilt werden; aus biologischer Sicht fehlen die Altersstadien jedoch weitgehend. Da multifunktionale Waldbewirtschaftung verschiedenen Zielen gerecht werden muss, sind neue Definitionen nötig, die auch ökologisch-biologische Aspekte einbringen. Eine Möglichkeit wäre, die klassische Definition nach oben zu erweitern, wie es zum Beispiel in einem Projekt der ETH Zürich zur Verbreitung seltener Baumarten auf der Alpennordseite der Fall war. ${ }^{2}$ Hier wurden zwei zusätzliche Entwicklungsstufen definiert: schwaches Altholz $\left(\mathrm{d}_{\mathrm{dom}} 60-100 \mathrm{~cm}\right)$ und starkes Altholz $\left(\mathrm{d}_{\mathrm{dom}}>100 \mathrm{~cm}\right)$.

Für das Aufspüren erhaltenswerter Altwälder und -bestände schlagen wir zehn qualitative Merkmale vor (Tabelle 1). Je mehr dieser Merkmale zutreffen, desto schützenswerter ist ein Wald. Quantitative Merkmale, von denen einige im nächsten Abschnitt besprochen werden, können die Erkennung von Altwäldern erleichtern.

\section{Quantitative Merkmale zum Erkennen von alten Wäldern in der Schweiz}

\section{Anzahl dicker Bäume}

Während im Schweizer Wald im Durchschnitt ein bis zwei Giganten (Bäume mit BHD >80 cm) pro Hektare stehen (Tabelle 3), sind in den Schweizer Naturwaldreservaten mit 1.4 bis 4.5 Giganten pro

\footnotetext{
1 Im LFI werden die Entwicklungsstufen Jungwuchs/Dickung $(<12 \mathrm{~cm})$, Stangenholz $(12-30 \mathrm{~cm})$, schwaches $(31-40 \mathrm{~cm})$, mittleres $(41-50 \mathrm{~cm})$ und starkes Baumholz $(>50 \mathrm{~cm})$ unterschieden.

2 www.wm.ethz.ch/sebapub/seba_castanea/SEBACASTANEA_ KA3_anleitung.pdf (14.1.2015)
} 


\begin{tabular}{|c|c|c|}
\hline Bezugseinheit & $\begin{array}{l}\text { Anzahl Giganten } \\
\text { pro Hektare }\end{array}$ & Quelle \\
\hline \multicolumn{3}{|l|}{ Europäische Ur- und Naturwälder } \\
\hline $\begin{array}{l}\text { Buchenwälder: La Tillaie, Le Gros Fouteau } \\
\text { (Frankreich), Serrahn (Deutschland), } \\
\text { Mirdita, Puka und Rajka (Albanien), } \\
\text { Uholka-Shyrokyj Luh (Ukraine) }\end{array}$ & $5-23$ & $\begin{array}{l}\text { Heiri et al (2011) } \\
\text { Commarmot et al } \\
(2013)\end{array}$ \\
\hline Fichten-Tannen-Buchenurwald (Slowakei) & 16 & \multirow[t]{3}{*}{ Heiri et al (2011) } \\
\hline Fichtenwälder (Westkarpaten) & $6-18(\geq 72 \mathrm{~cm})$ & \\
\hline $\begin{array}{l}\text { Linden-Hagebuchen-Eichen-Ulmen- } \\
\text { Mischwälder (Białowieża, Polen) }\end{array}$ & $5-17$ & \\
\hline Naturwälder Mitteleuropas & $10-17$ & Nilsson et al (2002) \\
\hline \multicolumn{3}{|l|}{ Naturwaldreservate der Schweiz } \\
\hline Nadelwälder & $3.6( \pm 1.1)$ & \multirow[t]{6}{*}{ Heiri et al (2011) } \\
\hline Buchenwälder & $1.4( \pm 0.6)$ & \\
\hline Übrige Laubwälder & $4.5( \pm 2.0)$ & \\
\hline Derborence (Wallis) & 9.3 & \\
\hline Scatlè (Graubünden) & 7.5 & \\
\hline Schweizerischer Nationalpark (Graubünden) & 1.3 & \\
\hline Leihubelwald (Obwalden) & 10 & Streit \& Heiri (2011) \\
\hline St-Jean (Bern) & 11 & $\begin{array}{l}\text { Hallenbarter } \\
\text { \& Brang (2011) }\end{array}$ \\
\hline \multicolumn{3}{|l|}{ Schweizer Wirtschaftswald } \\
\hline Subalpine Stufe & 2.2 & \multirow[t]{3}{*}{ Brändli (2010) } \\
\hline Montane Stufe & 1.3 & \\
\hline Kolline und submontane Stufe & 1.2 & \\
\hline
\end{tabular}

Tab 3 Anzahl Giganten (Bäume $>80 \mathrm{~cm}$ BHD) in mitteleuropäischen Naturwäldern, Schweizer Waldreservaten und Wirtschaftswäldern.

\begin{tabular}{|l|r|r|r|r|}
\hline \multirow{2}{*}{ Höhenstufe } & \multicolumn{4}{|c|}{ Anteil der Waldfläche mit letztem Eingriff vor $>$ 50 Jahren (\%) } \\
\cline { 2 - 5 } & $\mathbf{1 9 8 3 - 1 9 8 5}$ & $\mathbf{1 9 9 3 - 1 9 9 5}$ & $\mathbf{2 0 0 4 - 2 0 0 6}$ & $\mathbf{2 0 0 9 - 2 0 1 3}$ \\
\hline Obere subalpine & $33.3 \pm 4.1$ & $41.6 \pm 4.3$ & $59.6 \pm 4.2$ & $60.3 \pm 4.2$ \\
\hline Untere subalpine & $19.6 \pm 1.6$ & $20.5 \pm 1.6$ & $25.3 \pm 1.8$ & $26.0 \pm 1.8$ \\
\hline Obere montane & $13.6 \pm 1.3$ & $15.0 \pm 1.3$ & $16.3 \pm 1.4$ & $17.7 \pm 1.4$ \\
\hline Untere montane & $8.6 \pm 1.0$ & $8.4 \pm 1.0$ & $9.7 \pm 1.1$ & $11.6 \pm 1.2$ \\
\hline Kolline/submontane & $6.5 \pm 0.8$ & $6.2 \pm 0.8$ & $10.8 \pm 1.0$ & $11.1 \pm 1.0$ \\
\hline Schweiz & $12.5 \pm 0.6$ & $13.2 \pm 0.6$ & $16.9 \pm 0.6$ & $17.9 \pm 0.6$ \\
\hline
\end{tabular}

Tab 4 Anteil der Waldfläche, bei der der letzte Eingriff mehr als 50 Jahre zurückliegt, in Abhängigkeit der Höhenstufen. Mittelwert \pm Standardfehler. Quelle: LFI1-4

Hektare bereits einige mehr zu finden. Die Gigantendichte ist aber auch in den Reservaten noch wesentlich tiefer als in mitteleuropäischen Ur- und Naturwäldern, in denen pro Hektare 10 bis 17 solch dicker Bäume vorkommen. Nur in einzelnen Reservaten wie zum Beispiel in Derborence (Wallis) oder Scatlè (Graubünden) stehen bis zu elf Giganten pro Hektare.

Baumart und Wüchsigkeit des Standortes spielen eine grosse Rolle. Im seit über 100 Jahren ungenutzten Schweizerischen Nationalpark stehen zum Beispiel im Lärchen-Arvenwald erst 1.3 Giganten pro Hektare und im Bergföhrenwald gar keine (Tabelle 3). Diese subalpinen Wälder wachsen sehr langsam, und die Bäume auf schlechtwüchsigen Böden erreichen meist keine grossen Durchmesser. Oft kor- relieren die Durchmesser nicht mit dem Baumalter. Dies konnte auch im Bödmerenwald nachgewiesen werden (Horat et al 2005): Die dicksten Fichten sind nicht besonders alt, sondern wachsen auf den wüchsigeren Standorten, wo sie maximal 200 Jahre erreichen. Die Rekordträger (bis 484 Jahre) wachsen vielmehr auf sehr kargen Böden, die kaum noch waldfähig sind. Umgekehrt können zum Beispiel in intensiv bewirtschafteten Pappelkulturen auf geeigneten Standorten schon in 40 Jahren $80 \mathrm{~cm}$ dicke Bäume heranwachsen. Die Anzahl dicker Bäume kann also nicht als absolutes Mass zur Identifizierung alter Wälder gelten.

\section{Zeitdauer seit dem letzten Eingriff}

Das LFI4b (2009-2013; Abegg et al 2014) weist für 17.9\% der Schweizer Waldfläche einen Zeitpunkt von mehr als 50 Jahren seit des letzten Eingriffes aus (Tabelle 4), wobei die Unterschiede zwischen den Regionen gross sind. In der oberen subalpinen Stufe wurde in $60 \%$ der Wälder seit mehr als 50 Jahren nicht mehr eingegriffen, während dies in der kollinen und unteren montanen Stufe nur in 11\% der Wälder der Fall war. Allein genommen kennzeichnet dieses Merkmal jedoch noch nicht den Altwaldcharakter, denn auch nach mehreren Jahrzehnten Nutzungsaufgabe beispielsweise von ehemaligen Buchenniederwäldern oder Kastanienselven im Tessin sind diese Wälder im biologischen Sinne noch nicht alt.

\section{Bestandesalter und Entwicklungsstufe}

Auch das Bestandesalter und die Entwicklungsstufe, zwei weitere Merkmale des LFI, geben Hinweise auf das potenzielle Vorkommen von alten Wäldern oder Altwaldbeständen. Laut LFI4b (Abegg et al 2014) sind in der Schweiz 6.8\% der Wälder älter als geschätzte 160 Jahre (Tabelle 5), je nach Region sind es zwischen 1.1\% (Mittelland) und 13.4\% (Alpen). Alte Wälder finden sich also kaum im Mittelland und im Jura, viel häufiger jedoch in den Alpen. In einem Zeitraum von rund 30 Jahren, d.h. zwischen 1983-1985 und 2009-2013, hat die Waldfläche ohne Eingriff seit mehr als 50 Jahren in den oberen Lagen der Schweiz deutlich zugenommen, nämlich von $33.3 \%$ auf $60.3 \%$ in der oberen subalpinen Stufe und von $19.6 \%$ auf $26.0 \%$ in der unteren subalpinen Stufe (Tabelle 4). Trotzdem hat der Waldflächenanteil von über 160-jährigen Beständen in 20 Jahren, nämlich von 1993-1995 bis 20092013, in den Alpen von 20.9\% auf 13.4\% abgenommen (Tabelle 5). Eine mögliche Erklärung könnte die verstärkte Verjüngungstätigkeit in alten Beständen sein. Diese Zusammenhänge lassen vermuten, dass alte Wälder im ökologischen Sinn, trotz tendenziell weniger Eingriffen in höheren Lagen, sich wahrscheinlich flächenmässig im Rückgang befinden. Allerdings werden je nach Region im LFI4b zwischen 11 und 33\% der Waldbestände in der Schweiz als un- 


\begin{tabular}{|l|c|c|c|}
\hline \multirow{2}{*}{ Region } & \multicolumn{3}{|c|}{ Anteil der Waldfläche mit Bestandesalter >160 Jahre (\%) } \\
\cline { 2 - 4 } & $\mathbf{1 9 9 3 - 1 9 9 5}$ & $\mathbf{2 0 0 4 - 2 0 0 6}$ & $\mathbf{2 0 0 9 - 2 0 1 3}$ \\
\hline Jura & $4.1 \pm 0.9$ & $3.7 \pm 0.8$ & $2.8 \pm 0.7$ \\
\hline Mittelland & $0.8 \pm 0.3$ & $1.7 \pm 0.5$ & $1.1 \pm 0.4$ \\
\hline Voralpen & $5.3 \pm 0.9$ & $8.1 \pm 1.1$ & $6.0 \pm 1.0$ \\
\hline Alpen & $20.9 \pm 1.3$ & $15.6 \pm 1.2$ & $13.4 \pm 1.1$ \\
\hline Alpensüdseite & $6.2 \pm 1.3$ & $7.7 \pm 1.5$ & $8.3 \pm 1.5$ \\
\hline Schweiz & $8.8 \pm 0.5$ & $8.1 \pm 0.7$ & $6.8 \pm 0.5$ \\
\hline
\end{tabular}

Tab 5 Anteil der Waldfläche mit Bestandesalter $>160$ Jahre. Mittelwert \pm Standardfehler. Quelle: LF11-4

\begin{tabular}{|c|c|c|c|}
\hline \multirow[t]{2}{*}{ Baumart } & \multicolumn{2}{|r|}{ Urwald } & \multirow{2}{*}{$\begin{array}{c}\text { Schweizer Wirtschaftswald } \\
\text { Übliche Umtriebszeit } \\
\text { (Jahre) }\end{array}$} \\
\hline & $\begin{array}{l}\text { Alter } \\
\text { (Jahre) }\end{array}$ & Gebiet & \\
\hline \multirow[t]{4}{*}{ Tanne } & 600 & Rothwald, Österreich & \multirow[t]{4}{*}{$120-150$} \\
\hline & 450 & $\begin{array}{c}\text { Kubany, Tschechien, Dobroč, } \\
\text { Slowakei }\end{array}$ & \\
\hline & $350-400$ & Badín, Slowakei & \\
\hline & 350 & Perućica, Bosnia Herzegovina & \\
\hline \multirow[t]{5}{*}{ Fichte } & 500 & Slowenien & \multirow[t]{5}{*}{$120-150$} \\
\hline & 484 & Bödmerenwald, Schweiz & \\
\hline & 400 & Kubany, Tschechien & \\
\hline & 350 & Dobroč, Slowakei & \\
\hline & $300-650$ & Scatlè, Schweiz & \\
\hline \multirow[t]{6}{*}{ Buche } & $451\left(550^{*}\right)$ & Uholka, Ukraine & \multirow[t]{6}{*}{$120-160$} \\
\hline & 400 & Rothwald, Österreich & \\
\hline & $300-400$ & Kubany, Tschechien & \\
\hline & $200-250$ & Slowakei & \\
\hline & $>500$ & Perućica, Bosnia Herzegovina & \\
\hline & 503 & Apennin, Italien & \\
\hline Bergahorn & $250-270$ & Simonca, Slowakei & $80-100$ \\
\hline
\end{tabular}

Tab 6 Maximales Alter von Baumarten in verschiedenen Gebieten, ermittelt mit Jahrringzählung (resp. Schätzung [*]) an Urwaldriesen (nach Bigler 2003, Bütler et al 2011, Di Filippo et al 2012, Hillgarter 1971, Horat et al 2005, Nagel et al 2014 und Trotsiuk et al 2012). Zum Vergleich sind die im Schweizer Wald üblichen Umtriebszeiten gegenübergestellt.
Alter von Fichten auf circa 300 bis 650 Jahre geschätzt wurde (Hillgarter 1971). Bei Altersanalysen in Urwäldern hat sich gezeigt, dass die ältesten Bäume selten die dicksten sind und häufig auf kargen Kleinstandorten wachsen. Leider liegen in den wenigsten Fällen Daten zum Alter von Einzelbäumen vor. Altersanalysen werden meist nur für Spezialstudien gemacht. Sie sind jedoch eine gute Möglichkeit zum Nachweis echter Altwälder.

\section{Vorrat und Grundfläche}

Der Vorrat ist in alten Wäldern auch über grössere Flächen normalerweise sehr gross. Im Buchenurwald Uholka in den Karpaten beträgt der Lebendvorrat durchschnittlich $582 \mathrm{~m}^{3} /$ ha (Commarmot et al 2013), während in nordwest- und mitteleuropäischen Eichen- und Buchenwaldreservaten Vorräte von bis zu $869 \mathrm{~m}^{3} /$ ha gemessen wurden (Vandekerkhove et al 2009). In rumänischen Fichten-, Tannenund Buchenurwäldern wurden Durchschnittsvorräte von $587 \mathrm{~m}^{3} /$ ha gemessen, einzelne Bestände wiesen Hektarvorräte von bis zu $1195 \mathrm{~m}^{3}$ auf (Donita \& Dissescu 2001). Im Vergleich dazu steht im Schweizer Wald ein Vorrat von $350 \mathrm{~m}^{3} / \mathrm{ha}$, am höchsten sind die Werte in den Voralpen (448 m³/ha; LFI4b; Abegg et al 2014).

Auch die Grundfläche ist in alten Wäldern tendenziell sehr gross, zum Beispiel $36.6 \pm 0.8 \mathrm{~m}^{2} /$ ha im Buchenurwald Uholka-Shyrokyj Luh (Commarmot et al 2013) und 39-64 $\mathrm{m}^{2} /$ ha im Urwald Białowieża in Polen (Nilsson et al 2002). Gleichzeitig ist die Stammzahl eher niedrig. Schweizer Buchenwälder der Entwicklungsstufe «starkes Baumholz» $\left(\mathrm{d}_{\mathrm{dom}}>50 \mathrm{~cm}\right)$, obwohl in der waldbaulichen Definition als alt beurteilt, weisen grössere Stammzahlen (581 St./ha, Kluppschwelle bei $12 \mathrm{~cm}$ ) und eine kleinere Grundfläche $\left(31.5 \mathrm{~m}^{2} / \mathrm{ha}\right)$ auf als Buchenurwälder in Osteuropa (219-391 St./ha [trotz Kluppschwelle bei 8 und $7 \mathrm{~cm}$ ], $37.2-43.1 \mathrm{~m}^{2} / \mathrm{ha}$; Heiri et al 2012). Eine grosse Grundfläche zusammen mit einer kleinen Stammzahl ist charakteristisch für alte Wälder.

\section{Totholz}

Je nach Baumartenzusammensetzung und Entwicklungsphase sind in europäischen Urwäldern 40 bis $450 \mathrm{~m}^{3} /$ ha Totholz vorhanden. In sechs Schweizer Naturwaldreservaten wurde ein mittleres Totholzvolumen von $69 \mathrm{~m}^{3} / \mathrm{ha}\left(48-129 \mathrm{~m}^{3} / \mathrm{ha}\right)$ nachgewiesen (Herrmann et al 2012). Im Schweizer Wald gibt es laut LFI4b ungefähr $24 \mathrm{~m}^{3} / \mathrm{ha}$, wobei die Werte je nach Region stark schwanken (14-52 m³/ha). Liegt und steht mehr Totholz als durchschnittlich im Schweizer Wald, kann dies auf einen alten Wald hinweisen, muss aber nicht zwingend. Denn im Zuge von Stürmen können im Extremfall lokal sehr grosse Totholzmengen vorkommen, ohne dass jedoch noch alte Bäume stehen. Nur wenn auch die rat et al 2005), während in Scatlè (Graubünden) das 


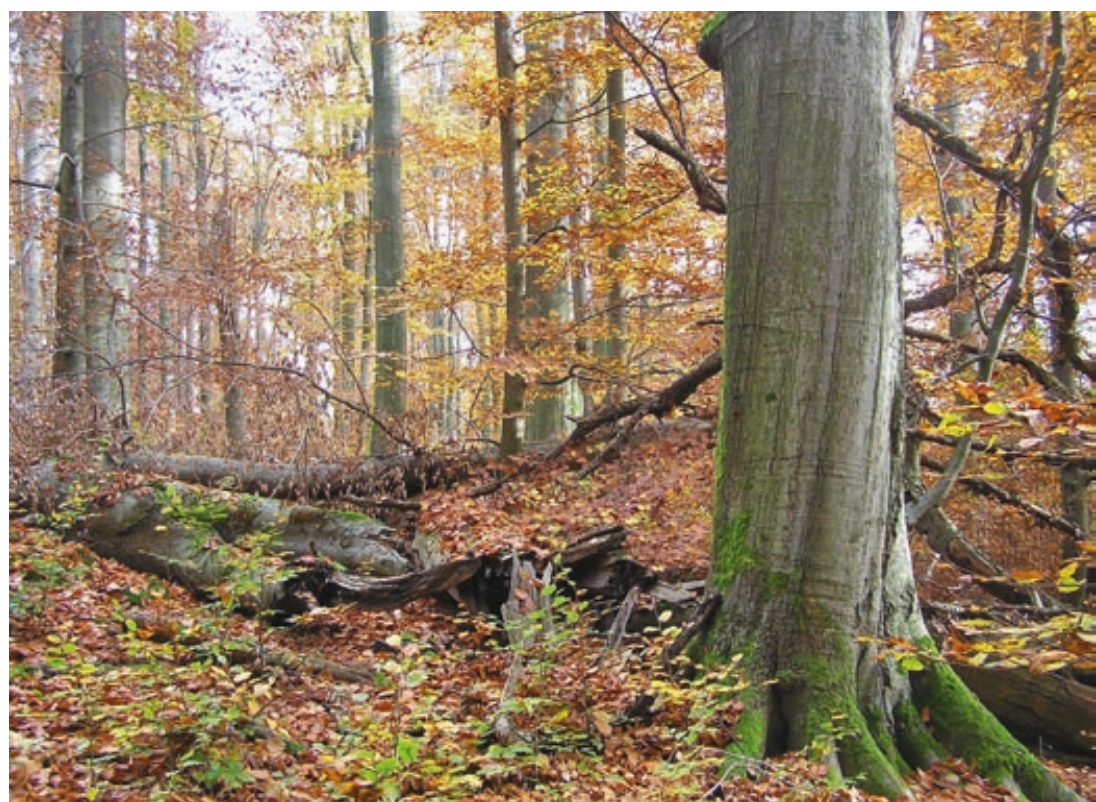

Abb 1 Buchen-Altwaldbestand.

fortgeschrittenen Abbaustadien des Holzes in naturnahen Anteilen vorhanden sind, hat man es mit grosser Wahrscheinlichkeit mit einem Altwald zu tun. Totholzvolumen in Altwäldern und Altwaldbeständen übersteigen die Mindestmengen - die sogenannten Schwellenwerte -, welche für spezialisierte Arten notwendig sind, und es sind insbesondere viele dicke tote Bäume vorhanden. Solche Schwellenwerte betragen ungefähr $20-30 \mathrm{~m}^{3} /$ ha für Nadelwälder, 30-40 m³/ha für montane Mischwälder und 30-50 m³ ha für kolline Eichen- oder Buchenwälder (Müller \& Bütler 2010).

\section{Ausblick}

Aufgrund ihrer Seltenheit und gleichzeitig grossen Bedeutung müssen alte Wälder und Bestände (Abbildung 1) als etwas Besonderes erkannt und entsprechend geschützt werden. Wir rufen alle Waldfachleute dazu auf, vorhandene Daten zu nutzen sowie Umfragen zu starten, um das Wissen über die Existenz, die Grösse und die Verteilung von Altwäldern lokal und regional zu vergrössern. Die in diesem Artikel besprochenen qualitativen und quantitativen Merkmale können zu ihrer Identifizierung dienen. Im Rahmen internationaler Prozesse und mit ihrer Biodiversitätsstrategie hat sich die Schweiz verpflichtet, bis 2020 eine ökologische Infrastruktur mit Schutz- und Vernetzungsflächen einzurichten (Schweizerischer Bundesrat 2012). Dazu gehören auch alte Wälder und Altwaldbestände. Die Erstellung eines landesweiten Inventars erhaltenswerter alter Wälder könnte dazu beitragen, das Bewusstsein über die Bedeutung alter Wälder und ihre Wertschätzung in Fachkreisen, bei Entscheidungsträgern und in der Bevölkerung zu verstärken. Alle, die für den Wald Verantwortung tragen, sollten letztlich zur
Überzeugung gelangen, dass die letzten Altwälder ein gewaltiges Naturerbe sind und zugleich ein riesiges Potenzial besitzen für künftige Herausforderungen, seien sie sozioökonomisch, zur Erkenntnisgewinnung für den Waldbau oder im Zusammenhang mit Klimafragen.

Eingereicht: 5. September 2014, akzeptiert (mit Review): 28. Januar 2015

\section{Literatur}

ABEGG M, BRÄNDLI UB, CIOLDI F, FISCHER C, HEROLD-BONARDI A ET AL (2014) Viertes Schweizerisches Landesforstinventar Ergebnistabellen und Karten im Internet zum LFI 2009-2013 (LFI4b). Birmensdorf: Eidgenöss Forsch.anst WSL. www.Ifi.ch/ resultate.

BIGLER C (2003) Growth-dependent tree mortality: ecological processes and modeling approaches based on tree-ring data. Zürich: ETH Zürich, Dissertation. 131 p.

BRÄNDLI UB, EDITOR (2010) Schweizerisches Landesforstinventar. Ergebnisse der dritten Erhebung 2004-2006. Birmensdorf: Eidgenöss Forsch.anstalt WSL. 312 p.

BRÄNDLI UB, ABEGG M, BÜTLER R (2011) Lebensraum-Hotspots für saproxylische Arten mittels LFI-Daten erkennen. Schweiz Z Forstwes 162: 312-325. doi: 10.3188/szf.2011.0312

BRANG P, HEIRI C, BUGMANN H, EDITORS (2011) Waldreservate. 50 Jahre natürliche Waldentwicklung in der Schweiz. Bern: Haupt. 271 p.

BÜTLER R, BOLLIGER M, SENN-IRLET B, WERMELINGER B (2011) Naturwälder als Lebensraum. In: Brang P, Heiri C, Bugmann $\mathrm{H}$, editors. Waldreservate: 50 Jahre natürliche Waldentwicklung in der Schweiz. Bern: Haupt. pp. 38-55.

COMMARMOT B, BRÄNDLI UB, HAMOR F, LAVNYY V, EDITORS (2013) Inventory of the largest primeval beech forest in Europe. A Swiss-Ukrainian scientific adventure. Birmensdorf: Swiss Federal Research Institute WSL. $69 \mathrm{p}$.

DI FILIPPO A, BIONDI F, MAUGERI M, SCHIRONE B, PIOVESAN G (2012) Bioclimate and growth history affect beech lifespan in the Italian Alps and Apennines. Glob Chang Biol 18: 960-972. DONITA N, DISSESCU R (2001) Historique des recherches concernant les forêts vierges. In: Giurgiu $V$ et al, editors. Les forêts vierges de Roumanie. Asbl Forêt wallone, Louvain-la-Neuve, Belgique. pp. 73-83.

EUROPEAN COMMUNITIES (2008) The economics of ecosystems and biodiversity. An interim report. Cambridge: TEEB. 70 p.

GABRIEL J, BRÄKER OU, MATTER JF (2001) Altersstruktur und Wachstum anhand geworfener Bäume auf einer Windwurffläche im Waldreservat Bödmeren. Schweiz Z Forstwes 152: 61-70. doi: 10.3188/szf.2001.0061

GÖTZ M (2001) Baumalterszusammensetzung und Absterbeprozesse in einer ausgewählten Teilfläche des Waldreservats Scatlè/Brigels. Zürich: ETH Zürich, Professur Waldbau, Diplomarbeit. $140 \mathrm{p}$.

HALLENBARTER D, BRANG P (2011) Baumriesen in St. Jean. In: Brang $\mathrm{P}$, Heiri $\mathrm{C}$, Bugmann $\mathrm{H}$, editors. Waldreservate. 50 Jahre natürliche Waldentwicklung in der Schweiz. Bern: Haupt. pp. 187-195.

HEIRI C, BRANG P, COMMARMOT B, MATTER JF, BUGMANN H (2011) Walddynamik in Schweizer Naturwaldreservaten: Kennzahlen und Trends. In: Brang P, Heiri C, Bugmann H, editors. Waldreservate. 50 Jahre natürliche Waldentwicklung in der Schweiz. Bern: Haupt. pp. 73-89.

HEIRI C, BRÄNDLI UB, BUGMANN H, BRANG H (2012) Sind Naturwaldreservate naturnäher als der Schweizer Wald? Schweiz Z Forstwes 163: 210-221. doi: 10.3188/szf.2012.0210

HERRMANN S, CONDER M, BRANG P (2012) Totholzvolumen und -qualität in ausgewählten Schweizer Naturwaldreservaten. Schweiz Z Forstwes 163: 222-231. doi: 10.3188/szf.2012.0222 
HILLGARTER FW (1971) Waldbauliche und ertragskundliche Untersuchungen im subalpinen Fichtenurwald Scatlè/Brigels. Zürich: ETH Zürich, Dissertation. 80 p.

HÖHN M, GUGERLI F, ABRAN P, BISZTRAY G, BUONAMICI A ET $A L$ (2009) Variation in the chloroplast DNA of Swiss stone pine (Pinus cembra L.) reflects contrasting post-glacial history of populations from the Carpathians and the Alps. J Biogeogr 36: 1798-1806.

HORAT S, LIECHTI T, RIGLING A (2005) Altersdifferenzierung der Bäume. In: Stiftung Urwaldreservat Bödmeren, editors. Urwaldcharakteristiken des Bödmerenwaldes. Ein interdisziplinäres Forschungsprojekt. Lenzburg: Burger \& Stocker. pp. 60-68.

JAKOBY O, RADEMACHER C, GRIMM V (2010) Modelling dead wood islands in European beech forests: how much and how reliably would they provide dead wood? Eur J For Res 129: 659-668.

KÄLIN W, EDITOR (1997) Urwald in den Schwyzer Bergen. Schöne wilde Bödmeren. Zürich: Werd. $136 \mathrm{p}$.

KORPEL' Š (1995) Die Urwälder der Westkarpaten. Stuttgart: Fischer. 310 p.

KÜHN S, ULLRICH B, KÜHN U (2002) Deutschlands alte Bäume. München: BLV. 159 p.

MÜHLETHALER B (2006) Heilmittelhersteller hilft Heilpflanze. Umwelt (3): 38-39.

MÜLLER J, BUSSLER H, BENSE U, BRUSTEL H, FLECHTNER G ET AL (2005) Urwald relict species - saproxylic beetles indicating structural qualities and habitat tradition. Waldökologie online 2: 106-113.

MÜLLER J, BÜTLER R (2010) A review of habitat thresholds for dead wood: a baseline for management recommendations in European forests. Europ J For Res 129: 981-992.

MÜLLER M, LACHAT T, BÜTLER R (2012) Wie gross sollen Altholzinseln sein? Schweiz Z Forstwes 163: 49-56. doi: 10.3188/ szf.2012.0049

NAGEL, T, SVOBODA, M, KOBAL M (2014) Disturbance, life history traits, and dynamics in an old-growth forest landscape of southeastern Europe. Ecol Appl 24: 663-679.

\section{Les vieilles forêts en Suisse, où se trouvent-elles?}

Les vieilles forêts et peuplements ont des valeurs multiples: biologique, scientifique, socio-économique, esthétique, spirituelle et culturelle. Elles sont rares en Europe centrale. En Suisse, aucune vue d'ensemble des vieilles forêts est disponible. Nous proposons une définition, à I'aide de dix critères qualitatifs, pour l'identification de tels objets méritant la protection: 1) âge du peuplement supérieur au temps de rotation normale, 2) grand volume sur pied, 3) structure hétérogène avec présence de stades de développement tardifs; 4) libre déroulement des perturbations et processus naturels, 5) grands volumes de bois mort (au minimum $30 \mathrm{~m}^{3} / \mathrm{ha}$ ) avec dimensions et degrés de décomposition divers, 6) essences autochtones, 7) cortèges d'espèces naturels, 8) dernière exploitation datant d'au moins 50 ans ou seulement utilisation extensive depuis, 9) longue continuité du couvert forestier, 10) surface minimale d'environ 30 ha pour les vieilles forêts et de 1 ha pour les vieux peuplements. Lors de la recherche des vieilles forêts, les critères quantitatifs de l'Inventaire forestier national et d'autres sources sont également utiles. Nous faisons appel à tous les professionnels de la forêt pour l'identification des vieilles forêts dignes de protection, pour l'information de la population et des décideurs quant à leur valeur intrinsèque, dans le but de protéger cet héritage naturel unique de façon appropriée.
NILSSON SG, NIKLASSON M, HEDIN J, ARONSSON G, GUTOWSKI JM ET AL (2002) Densities of large living and dead trees in oldgrowth temperate and boreal forests. For Ecol Manage 161: 189-204.

PARVIAINEN J (2005) Virgin and natural forests in the temperate zone of Europe. For Snow Landsc Res 79: 9-18.

SCHEIDEGGER C, STOFER S (2015) Bedeutung alter Wälder für Flechten: Schlüsselstrukturen, Vernetzung, ökologische Kontinuität. Schweiz Z Forstwes 166: 75-82. doi: 10.3188/ szf. 2015.0075

SCHÜTZ JP (2003) Die Prinzipien der Waldnutzung und der Waldbehandlung. Zürich: ETH Zürich, Skript Vorlesung Waldbau I. $212 \mathrm{p}$.

SCHWEIZERISCHER BUNDESRAT (2012A) Strategie Biodiversität Schweiz vom 25. April 2012. BBI 2012: 7239-7342.

STIFTUNG URWALDRESERVAT BÖDMEREN, EDITOR (2005) Urwaldcharakteristiken des Bödmerenwaldes. Ein interdisziplinäres Forschungsprojekt. Lenzburg: Burger \& Stocker.143 p.

STREIT K, HEIRI C (2011) Die Tanne auf dem Vormarsch im Leihubelwald. In: Brang P, Heiri C, Bugmann H, editors. Waldreservate. 50 Jahre natürliche Waldentwicklung in der Schweiz. Bern: Haupt. pp. 175-185.

TROTSIUK V, HOBI M, COMMARMOT B (2012) Age structure and disturbance dynamics of the relic virgin beech forest Uholka (Ukrainian Carpathians). For Ecol Manage 265: 181-190.

VANDEKERKHOVE K, DE KEERSMAEKER L, MENKE N, MEYER P, VERSCHELDE $P$ (2009) When nature takes over from man: Dead wood accumulation in previously managed oak and beech woodlands in North-western and Central Europe. For Ecol Manage 258: 425-435.

WINTER S, LÜDERITZ M, RZANNY, M (2015) Unterirdische Kontinuität und Pilzvielfalt alter Waldstandorte. Schweiz Z Forstwes 166: 83-90. doi: 10.3188/szf.2015.0083

WIRTH C, MESSIER C, BERGERON Y, FRANK D, FANKHÄNEL A (2009) Old-growth forest definitons: a pragmatic view. In: Wirth C, Gleixner G, Heimann M, editors. Old-growth forests. Function, fate and value. Berlin: Springer, Ecological Studies 207. pp. 11-33.

\section{The search for old-growth forests in Switzerland}

Old-growth forests and stands have multiple values, such as biological, scientific, socioeconomic, aesthetic, spiritual and cultural ones, but they are very rare in central Europe. Currently, an overview about the existence and size of old-growth forest patches in Switzerland is missing. We propose a definition for old-growth forests worth preserving based on ten criteria: 1) presence of veteran trees, 2) significant biomass, 3) heterogeneous stand structure including senescent and decaying stages, 4) presence of natural processes and disturbances, 5) high dead wood volume (at least $30 \mathrm{~m}^{3} / \mathrm{ha}$ ) and a large diversity of dimensions and decay stages of dead wood pieces, 6) autochthonous tree species, 7) diverse and natural species assemblages, 8) only extensive or no harvesting for the last 50 years, 9) long continuity of forest cover, 10) minimal area of 30 ha for old-growth forests and 1 ha for oldgrowth stands. When searching for old-growth forests and stands worth preserving, quantitative criteria from the $\mathrm{Na}$ tional Forest Inventory and other sources should also be used. We call on all forest specialists to identify old-growth patches, to inform the population and decision makers about their high value, and to protect them in an adequate manner as a unique natural heritage. 